STUDI

FRANCESI

\section{Studi Francesi}

Rivista quadrimestrale fondata da Franco Simone

181 (LXI | I) | 2017

PASCAL QUIGNARD: LES "PETITS TRAITES" AU FIL DE

LA RELECTURE - sous la direction de Mireille Calle-

Gruber, Stefano Genetti et Chantal Lapeyre

\title{
La Grande Guerre des écrivains, R. Vignest, J.-N. Corvisier (éds.)
}

\section{Margareth Amatulli}

\section{QpenEdition \\ Journals}

Edizione digitale

URL: http://journals.openedition.org/studifrancesi/6916

DOI: $10.4000 /$ studifrancesi.6916

ISSN: 2421-5856

Editore

Rosenberg \& Sellier

Edizione cartacea

Data di pubblicazione: 1 marzo 2017

Paginazione: 175-176

ISSN: 0039-2944

Notizia bibliografica digitale

Margareth Amatulli, « La Grande Guerre des écrivains, R. Vignest, J.-N. Corvisier (éds.) », Studi Francesi [Online], 181 (LXI | I) | 2017, online dal 01 avril 2017, consultato il 18 septembre 2020. URL : http:// journals.openedition.org/studifrancesi/6916 ; DOI : https://doi.org/10.4000/studifrancesi.6916

Questo documento è stato generato automaticamente il 18 settembre 2020.

\section{c) (†)}

Studi Francesi è distribuita con Licenza Creative Commons Attribuzione - Non commerciale - Non opere derivate 4.0 Internazionale. 


\title{
La Grande Guerre des écrivains, $\mathrm{R}$. Vignest, J.-N. Corvisier (éds.)
}

\author{
Margareth Amatulli
}

\section{NOTIZIA}

La Grande Guerre des écrivains, sous la direction de Romain VIGNEST et Jean-Nicolas CORVISIER, Paris, Classiques Garnier, 2015, «Rencontres», 781 pp.

1 I trentasei contributi che costituiscono questa copiosa miscellanea offrono una vasta panoramica della ricerca sulla Grande Guerra e del suo impatto ideologico ed estetico sulle letterature e sulle opere cinematografiche. Di stampo variegato, firmati da studiosi di diversa provenienza, gli articoli, preceduti da una breve presentazione e da una ricca introduzione storica, delineano un itinerario cronologico suddiviso in due parti che percorre la Francia dal 1914 al 2013 prima di rivolgere lo sguardo, nella terza parte del volume, al di là dei confini francesi. Come si deduce dal titolo della prima parte, «En guerre (1914-1918)», i primi diciannove saggi qui inclusi convergono sugli autori che hanno fatto esperienza più o meno diretta del campo di battaglia: dai più celebri, come Péguy (J.-P. Rioux, Charles Péguy en pantalon rouge, pp. 35-48), Claudel (D. ALEXANDRE, Paul Claudel en guerre (1914-1918), pp.49-70), Apollinaire (L. Michel, Le Patriotisme d'Apollinaire, pp. 203-222) - dei quali viene esplorata la dimensione patriottica spesso oggetto di controversie - ai più discussi, come il nazionalista Barrès (V. RAMBAUD, Maurice Barrès. Une parole qui ne passe pas, pp. 71-84) e il pacifista Rolland (R. RoudiL, La Mêlée des nations. Romain Rolland et «L'Âge de la haine» (1914-1915), pp. 85-106). Tra gli autori noti ritornano i nomi di Céline, lo scrittore su cui la guerra ha inciso in modo prolungato e deciso, come dimostra Henri gODARD (Céline. Au commencement était la guerre, pp. 325-330), di Gide, per il quale il conflitto è l'occasione di una rigenerazione morale della Francia (P. MASson, André Gide et la Grande Guerre, pp.129-148), di Apollinaire che l'ha tradotta in un'estetizzazione erotica (J.-Y. CASANOVA, Guillaume Apollinaire. Portrait du poète en Éros guerrier, pp. 181-202). Da tema di 
scrittura, la guerra può farsi discorso declinabile in vari modi: in Cendrars copre trent'anni di vita e si evolve attraverso le opere di poesia, di testimonianza e di finzione (M. TOURET, Blaise Cendrars. Une guerre de trente ans, pp. 223-246), mentre nell'opera di Aragon è contenuto in modo criptico ma non per questo meno espressivo (0. BARBARANT, Le Dire indirect. Aragon «classe 17» et après, pp. 247-270) e in Giono emerge in prossimità del secondo conflitto dopo anni di silenzio (H. SolnicA, La Description de la guerre par Jean Giono dans "Le grand troupeau". Dire ce que nul ne peut entendre, pp. 305-324).

2 Agli autori celebri si affiancano scrittori meno conosciuti dal grande pubblico e cui il volume rende implicitamente omaggio: l'antimilitarista Léautaud rimasto volontariamente "fuori dalla mischia" (M. KANTOROW, Paul Léautaud, antipatriote «en dehors de la mêlée», pp.107-128), il padre gesuita Teilhard de Chardin che riporta l'esperienza umana e spirituale da lui vissuta durante il conflitto (J.-D. BEAUDIN, Pierre Teilhard de Chardin dans la guerre, pp.149-158), il medico Duhamel, che tratteggia il quadro delle sofferenze dei malati (E. GIRARD, Au front. Un médecin injustement oublié, Georges Duhamel, pp.159-180), l'ex combattente Dorgelès che offre una visione retrospettiva della guerra ("Les Croix de bois" de Roger Dorgelès. Une écriture de la rétrospection, pp. 271-304). Testi di carattere generale affiancano, quindi, analisi di singole opere. M. DAMBRE, ad esempio, esplora l'entusiasmo guerriero di Drieu la Rochelle contenuto nelle Interrogations ("Interrogations" de Drieu la Rochelle, pp. 331-346), mentre Georgette WACHTEL si concentra sulla descrizione realistica della vita delle trincee riportata da Barbusse in Le feu (Henri Barbusse. Un pacifiste en guerre, pp. 347-366) e Cécilia suzzoni individua il nesso profondo che unisce La Campagne avec Thucydide di Thibaudet all'opera dello storico greco (Albert Thibaudet, "La Campagne avec Thucydide", pp. 367-382). La prima parte del volume si chiude con un articolo sulle fonti della storia che non possono prescindere da quelle meno ufficiali come Les Lettres de Poilus, dal nome con cui venivano definiti i soldati francesi durante la prima guerra mondiale (S. NOURRY-NAMUR, La République, l'école et les poilus..., pp. 382-411).

3 I dieci interventi contenuti nella seconda parte del volume intitolata «L'Empreinte (1918-2013)» esaminano l'impatto della guerra sulle avanguardie (M. MURAT, Les Avantgardes littéraires et la Grande Guerre, pp.415-434), sulla letteratura popolare (J.-N. CORVISIER e G. TALON, La Grande Guerre dans la littérature populaire est dans le roman d'aventure et d'évasion, pp. 481-510), sul romanzo francese degli anni '19-'29 (G. TALON, Guerre et révolution dans le romans français (1919-1929), pp. 473-480) e, più in generale, sulle delusioni del progresso espresse da Aragon, Drieu la Rochelle e Malraux (J.-L. LOUBET DEL BAYLE, La Guerre de 14. Des écrivains et les désillusion du progrès, pp. 565-580). Come nella prima parte del volume, lo sguardo panoramico di questi contributi non esclude la lettura di singole opere a firma di Martin du Gard, Rolland e Confiant (M. GRANGE, "L'été 1914" de Roger Martin du Gard. Le récit d'un engrenage implacable, pp. 443-458; O. RonY, L'Ombre portée de la guerre de 1914 sur les derniers tomes des "Hommes de bonne volonté", pp. 459-472; R. VIGNEST, «Là-bas", la "mère patrie". Amour et dépit dans "Le Bataillon créole" de Raphaël Confiant, pp. 525-542). Gli autori noti ritornano nei saggi dedicati a Proust, nel cui comandante Duroc evocato in Le Côté de Guermantes Luc FRAISSE intravede il modello del generale Foch (Proust historien et romancier de la Grande Guerre. Ce que le "commandant Duroc" doit à Ferdinand Foch, pp. 435-442), a Camus, che evoca la guerra nell'opera incompleta Le Premier homme, associandola emotivamente e simbolicamente alla figura paterna (Hèléne RUFAT, Albert Camus présente Jacques Corney. 
Un orphelin de la Grande Guerre, pp.511-524), a Valéry, cui il primo conflitto armato rivela l'impotenza di ogni forma di conoscenza (P. SIGNORILE, Paul Valéry, regards sur la guerre, pp. 543-564).

L'ultima parte del volume «Regards étrangers» attraversa la prima guerra mondiale nell'immaginario magrebino (G. DUGAS, La grande guerre dans l'imaginaire maghrébin. Trois cuvres d'expression françaises et leur destinée, pp. 609-618), italiano (I. VIOLANTE, Lambeaux de murs et de mots. La Grande Guerre de Giuseppe Ungaretti, pp. 663-682), francese e tedesco (G. WACHTEL, Trois écrivains de la Grande Guerre, trois regards, trois voix, pp.583-607), inglese (D. DÉLAS, Wilfred Owen (1883-1918). Le lyrisme à l'épreuve du carnage, pp. 619-642), americano (A. GuILLAIN, William Faulkner. La Grande Guerre et la fabrique du héros détraqué, pp. 643-662) e serbo (M. SREBRO, «Le Golgotha albanais» ou la traversée des Enfers. L'écho de la Grande Guerre dans le roman serbe, pp. 683-714).

5 Il capitolo conclusivo della miscellanea "prolunga" la riflessione verso altri media e indaga sulla rappresentazione cinematografica della Grande Guerra nel tempo, sul suo rapporto con il testo letterario e sui generi e gli stili che sperimenta (M. SERCEAU, Prolongement. La Grande Guerre, de la littérature au cinéma, pp. 715-741).

6 Nell'evidente disparità di intenti e di competenze, i contributi di giovani ricercatori e docenti delle scuole superiori e dell'università che formano il volume concorrono alla lettura della Prima guerra mondiale nel suo primo centenario rivolgendosi sia al lettore curioso che a quello più esperto. 